\title{
Workshop on Graph-Based Tools
}

\author{
Tom Mens, Andy Schürr and Gabriele Taentzer \\ Université de Mons-Hainaut, Belgium tom.mens@umh.ac.be \\ Technische Universität Darmstadt, Germany \\ andy.schuerr@es.tu-darmstadt.de \\ Technische Universität Berlin, Germany \\ gabi@cs.tu-berlin.de
}

\begin{abstract}
Graphs are well-known, well-understood, and frequently used means to depict networks of related items. They are successfully used as the underlying mathematical concept in various application domains. In all these domains, tools are developed that store, retrieve, manipulate and display graphs. It is the purpose of this workshop to summarize the state of the art of graph-based tool development, bring together developers of graph-based tools in different application fields and to encourage new tool development cooperations.
\end{abstract}

\section{Motivation}

Graphs are an obvious means to describe structural aspects in various fields of computer science. They have been successfully used in application areas such as compiler compiler toolkits, constraint solving problems, generation of CASE tools, model-driven software development, pattern recognition techniques, program analysis, software engineering, software evolution, software visualization and animation, and visual languages. In all these areas, tools have been developed that use graphs as an important underlying data structure. Since graphs are a very general structure concept, still novel application areas emerge which are of interest.

It is a challenge to handle graphs in an effective way. Using graphs inside tools, the following topics play an important role: efficient graph algorithms, empirical and experimental results on the scalability of graphs, reusable graphmanipulating software components, software architectures and frameworks for graph-based tools, standard data exchange formats for graphs, more general graph-based tool integration techniques, and meta CASE tools or generators for graph-based tools. The aim of the Second Workshop on Graph-Based Tools (GraBaTs) is to bring together developers of all kinds of graph-based tools in order to exchange their experiences, problems, and solutions concerning the efficient handling of graphs. The first workshop on this topic [5] took place two years ago as a satellite event of the 1 . Int. Conference of Graph Transformation (ICGT) [2].

The GraBaTs workshop is of special relevance for the conference on graph transformation: In many cases, the application of graph transformation technology requires the existence of reliable, user-friendly and efficiently working graph 
transformation tools. These tools in turn have to be built on top of basic services or frameworks for graphs which are the main topic of our workshop.

\section{Workshop Issues}

The workshop aims at bringing together tool developers from different fields, dealing with graphs from different perspectives. In the following, we give an overview on the most important perspectives.

\subsection{Graph-Based Tools for Visual Modelling Techniques}

Nowadays it is well accepted to use graphs or graph-like structures to describe the underlying structures of visual modelling techniques. The European Research Training Network SegraVis [8] is dedicated to develop new solutions for the definition and implementation of visual modelling languages. Mainly, graph transformation [3] and meta-modelling approaches [6] use graphs to define visual modelling languages. For a model driven development, especially model transformations are needed, either to validate models or to translate them into executable code.

Especially, graph-based tools are considered for generating a modelling environment for a sub-language of the UML [9] containing at least a reasonable variant of Statechart Diagrams. Although this modelling technique is still graphlike, it contains already a number of typical visual concepts and serves, thus, very well as reference application. Contributions to this topic are meant to continue the discussion and comparison of visual language definition started at the "Statechart contest" at VLFM'01 [10]. This time, the emphasis is laid on graph-based approaches and tool support on one hand, and the discussion is extended with regard to different semantics and transformations on the other hand.

\subsection{Graph Transformation}

Being a satellite event of the international conference on graph transformation, this workshop puts a special emphasis on graph transformation tools. The kind of graphs used by these tools and also their rules, the basic means to manipulate graphs, differ w.r.t. to the formal definition of their semantics, the way how occurrences (matches) are searched for, and how matching rules are applied eventually $[7,3,2]$.

In tools, graph transformation is mainly applied to visual languages, model transformation, specification, code generation, verification, restructuring, evolution and programming of software systems. Developers of graph transformation tools may profit from other workshop participants concerning more efficient realizations of basic functionality, while developers of other graph-based tools might find the graph transformation paradigm attractive to implement certain graph manipulations. The workshop may also provide insights to apply these tools to other application domains. 


\subsection{Common Exchange Formats for Graphs}

To support interoperability between various graph-based tools, several initiatives on the development of common exchange formats for graphs have been founded. These formats are all based on the extensible markup language XML developed to interchange documents of arbitrary types. While the graph drawing community favors a format called GraphML [4], preceding discussions at several workshops of the re-engineering and graph transformation community led to the format GXL [1]. Another topic of interest in this direction is an exchange format for graph transformation systems called GTXL which is under development and which will be built on top of GXL.

\section{Workshop Organization}

The program committee of this workshop consists of Luciano Baresi, Ulrik Brandes, Holger Giese, Gabor Karsai, Scott Marshall, Mark Minas, Tom Mens, Andy Schürr, Gabriele Taentzer, Daniel Varro, Pieter Van Gorp, Andreas Winter, Albert Zündorf. Altogether, 20 papers have been submitted for GraBaTs. The workshop is planned for one day. More information about the workshop including its program and an electronic version of all accepted papers appearing in the electronic notes of Theoretical Computer Science (ENTCS) can be found on the workshop web page: http://tfs.cs.tu-berlin.de/grabats

\section{References}

1. GXL http://www. gupro. de/GXL, 2002.

2. A. Corradini, H. Ehrig, H.-J. Kreowski, and G. Rozenberg, editors. Graph Transformation, 1st Int. Conference, volume 2505. Springer LNCS, 2002.

3. H. Ehrig, G. Engels, H.-J. Kreowski, and G. Rozenberg, editors. Handbook on Graph Grammars and Computing by Graph Transformation: Applications, Languages, and Tools, volume 2. World Scientific, Singapore, 1999.

4. The GraphML File Format, 2004. Available at http://graphml.graphdrawing. org.

5. T. Mens, A. Schürr, and G. Taentzer, editors. Graph-Based Tools (GraBaTs'02), volume 72. Electronic Notes in Theoretical Computer Science (ENTCS), 2002.

6. Meta Object Facilities - version 1.4, 2002. Available at http://www.omg.org/ technology/documents/formal/mof .htm.

7. G. Rozenberg, editor. Handbook of Graph Grammars and Computing by Graph Transformations, Volume 1: Foundations. World Scientific, 1997.

8. Syntactic and Semantic Integration of Visual Modeling Techniques, 2004. Available at http://www.segravis .org.

9. Unified Modeling Language - version 1.4, 2002. Available at http://www.omg. org/technology/documents/formal/uml.htm.

10. Symposium on Visual Languages and Formal Methods - Statecharts Modeling Contest, 2004. Available at http://www2-data.informatik. unibw-muenchen.de/ VLFM01/Statecharts. 